\title{
Scalable Compact Models for Fast Design Optimization of Complex Electromagnetic Systems
}

\author{
Francesco Ferranti*, Tom Dhaene*, \\ Luc Knockaert*, Giulio Antonini ${ }^{+}$, Antonio Ciccomancini Scogna $^{\ddagger}$ \\ *Department of Information Technology, Ghent University-IBBT, Gaston Crommenlaan 8 Bus \\ 201, B-9050, Ghent, Belgium \\ Email: francesco.ferranti@intec.ugent.be; tom.dhaene@intec.ugent.be; \\ luc.knockaert@intec.ugent.be \\ ${ }^{+}$Dipartimento di Ingegneria Elettrica e dell'Informazione, Università degli Studi dell'Aquila, \\ 67100, L'Aquila, Italy \\ Email: giulio.antonini@univaq.it \\ ${ }^{\ddagger}$ CST of America, 492 Old Connecticut Path, Suite 505, Framingham, MA, 01701, USA \\ E-mail: Antonio.Ciccomancini@cst.com.
}

\begin{abstract}
We propose a new parametric macromodeling technique for complex electromagnetic systems described by scattering parameters, which are parameterized by multiple design variables such as layout or substrate feature. The proposed technique is based on an efficient and reliable combination of rational identification, a procedure to find scaling and frequency shifting system coefficients, and positive interpolation schemes. Parametric macromodels can be used for efficient and accurate design space exploration and optimization. A design optimization example for a complex electromagnetic system is used to validate the proposed parametric macromodeling technique in a practical design process flow.
\end{abstract}

Keywords: parametric macromodeling, rational approximation, interpolation, design optimization, complex systems.

\section{Introduction}

Robust computer-aided design flows are essential for the minimization of signal integrity and electromagnetic (EM) compatibility issues in the development of modern digital and mixed-signal systems [1-5]. Chip packages, data busses, connectors, and cables require to be properly designed, since from the early design stage they can seriously limit transmission data rates or increase the bit error rate beyond acceptable limits. In order to avoid these problems, design space exploration, design optimization and sensitivity analysis are usually performed by multiple frequency-domain simulations for different design parameter values (e.g. layout features), trying to meet government regulations and customer requirements. In this perspective, parametric (scalable) macromodels are well suited to efficiently and accurately perform these design activities, while using multiple EM simulations is often high computationally expensive due to the high computational cost per simulation. Parametric macromodels are multivariate models that capture the complex behavior of 
EM systems, which is typically characterized by the frequency (or time) and several design parameters, such as layout or substrate features.

Over the last years, several different parametric macromodeling techniques have been developed. In [6], [7], both poles and residues are parameterized and it results in accurately modeling dynamic multivariate data. Overall stability and passivity of parametric macromodels are not guaranteed. Recently, some parametric macromodeling techniques able to guarantee overall stability and passivity of parametric macromodels have been proposed [8]-[11]. The techniques described in [8], [9] are based on the passive interpolation of a set of stable and passive univariate macromodels, called root macromodels, treated as input-output systems. This interpolation process of input-output systems leads to parameterize only the residues. A passive interpolation of the state-space matrices of a set of root macromodels is proposed in [10], [11], which provides an increased modeling capability with respect to [8], [9] due to the parameterization of both poles and residues. Unfortunately, these methods are sensitive to some issues related to the interpolation of state-space matrices [12] and can only deal with rational models of the same order. A passivity preserving interpolation of state-space matrices is performed through the matrix solution of positive-real and bounded-real lemma. It can be carried out using Linear Matrix Inequalities (LMI) or Riccati equation solvers. The computational complexity of a Riccati equation is $\mathrm{O}\left(n^{3}\right)$, while the cost of solving an equivalent $\mathrm{LMI}$ is $\mathrm{O}\left(n^{6}\right)$ [13], where $n$ is equal to the number of states. This high complexity prevents LMI-based techniques to be adopted for large scale problems.

This paper presents a new parametric macromodeling method for linear electromagnetic systems represented by the scattering (S) parameters, which indirectly parameterizes poles and residues, while guaranteeing overall stability and passivity of the resulting parametric macromodel. Initially, a set of univariate frequency-dependent macromodels related to different values of the design variables, called root macromodels [8], [9], is built by means of the Vector Fitting (VF) technique [14]. Stability for each root macromodel is enforced by pole-flipping [14], while passivity is checked and enforced by means of standard techniques (see e.g. [15], [16]). Next, the computation and parameterization of scaling and frequency shifting system coefficients for each root macromodel is performed. Finally, a parametric macromodel is obtained by a combination of root macromodels and corresponding scaling and frequency shifting coefficients, using positive interpolation schemes that preserve stability and passivity over the complete design space. The proposed technique has some significant advantages with respect to the existing ones: it can deal with root macromodels of different orders, it is able to guarantee overall passivity and stability without solving positive-real and bounded-real lemma and is not sensitive to the well-known issues related to the interpolation of state-space matrices. Hence, the proposed method is ready to be used in the optimization process of complex electromagnetic systems; the numerical results show that the parametric macromodel allows significant speed-ups with respect to full-wave EM simulations and, thus, it is well suited to be applied to a real design process flow.

\section{Parametric Macromodeling}

The proposed technique is able to build a parametric macromodel $\mathbf{R}(s, \boldsymbol{g})$ that accurately describes a set of data samples $\left\{(s, \boldsymbol{g})_{k}, \mathbf{H}(s, \boldsymbol{g})_{k}\right\}_{k=1}^{K_{t o t}}$, which depend on the complex frequency $s=j \omega$ and several design variables $\boldsymbol{g}=\left(g^{(m)}\right)_{m=1}^{M}$, such as layout and substrate features. Stability 
and passivity are preserved over the entire design space of interest. The proposed technique computes a parametric macromodel in the form

$$
\mathbf{R}(s, \boldsymbol{g})=\mathbf{C}(\boldsymbol{g})(s \mathbf{I}-\mathbf{A}(\boldsymbol{g}))^{-1} \mathbf{B}(\boldsymbol{g})+\mathbf{D}(\boldsymbol{g})
$$

Two data grids are used in the modeling process: an estimation grid and a validation grid. The estimation grid is utilized to build the root macromodels. The validation grid is used to validate the modeling capability of the parametric macromodel in a set of points of the design space previously not used for the construction of the root macromodels. The design space $\mathcal{D}(\boldsymbol{g})$ contains all parameters $\boldsymbol{g}$. To clarify the use of these two design space grids, we show in Fig. 1 a possible estimation and validation design space grid in the case of two design parameters $\boldsymbol{g}=\left(g^{(1)}, g^{(2)}\right)$. A root macromodel is built for each red $(x)$ point in the design space. The set of root macromodels is interpolated, as explained in what follows, to build a parametric model that is evaluated and compared with original data related to the blue (o) design space points. We note that these blue (o) points are not used for the generation of the root macromodels.

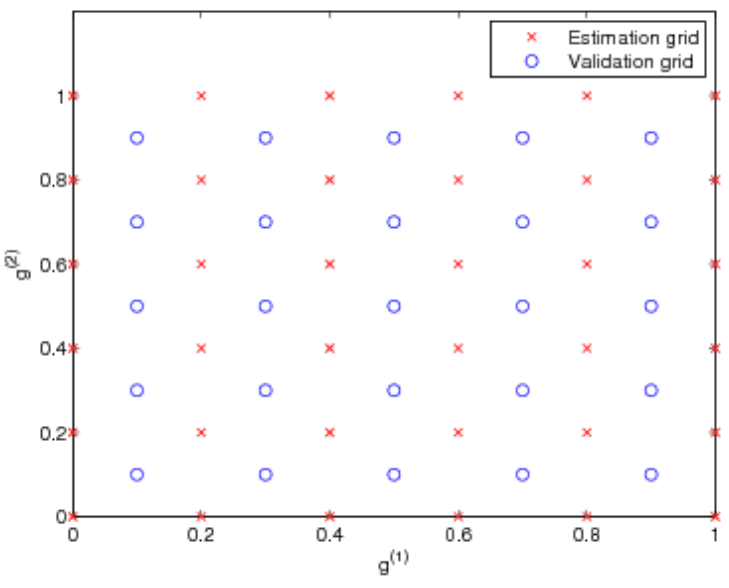

Figure 1: An example of estimation and validation design space grid.

$\mathrm{N}$-dimensional and scattered design space grids can also be treated by the proposed technique that does not impose any constraint on the number of design parameters and the distribution of root macromodels in the design space.

\subsection{Root Macromodels}

A set of frequency-dependent rational macromodels is built in the estimation design space grid by means of the VF technique [14], using a set of data samples $\left\{(s, \boldsymbol{g})_{k}, \mathbf{H}(s, \boldsymbol{g})_{k}\right\}_{k=1}^{K_{t o t}}$. A poleflipping scheme is used to enforce stability [14], while passivity assessment and enforcement can be accomplished using the robust standard techniques $[15,16]$. A set of stable and passive rational univariate macromodels that we call root macromodels is obtained in this first step.

\subsection{Scaling and Frequency Shifting Coefficients}


Once the root macromodels are computed, the next step is building a multivariate representation $\mathbf{R}(s, \boldsymbol{g})$. The design space is divided into cells using hyperrectangles (regular grid) [17] or simplices (regular and scattered grid) [18,19]. Figure 2 shows a possible 2-D design space divided into cells, in the regular and scattered case, respectively.
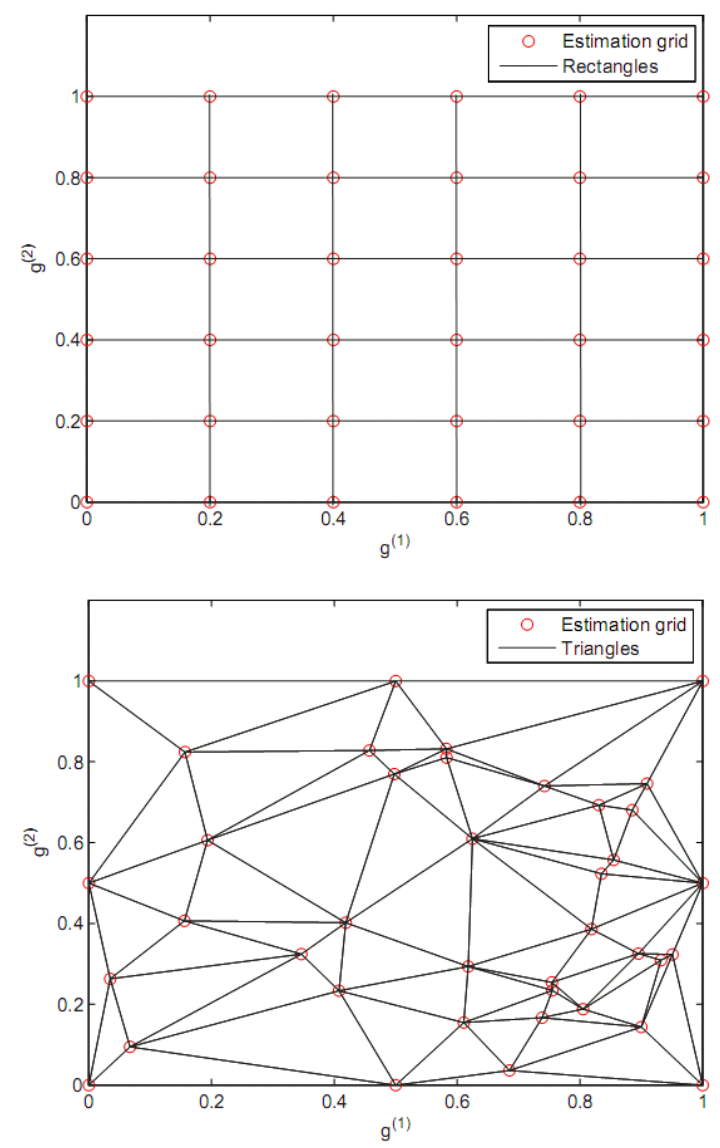

Figure 2: Design space divided into cells: regular (top) and scattered (bottom).

Once the design space is divided into cells, a local parametric model is associated to every cell that is a subdomain of the entire design space. A cell region of the design space is denoted as $\Omega_{i}, i=1, \ldots, P$ and the corresponding vertices as $\boldsymbol{g}_{k}^{\Omega_{i}}, k=1, \ldots, Q$. We note that each vertex corresponds to a root macromodel $\mathbf{R}\left(s, \boldsymbol{g}_{k}^{\Omega_{i}}\right)$. Scaling and frequency shifting system coefficients are found for each cell using an optimization procedure, so that they make each vertex an accurate approximant of the other cell vertices. For each vertex $\mathbf{R}\left(s, \boldsymbol{g}_{k}^{\Omega_{i}}\right)$, a set of scaling $\alpha_{1, k}\left(\boldsymbol{g}_{j}^{\Omega_{i}}\right), j=$ $1, \ldots, Q$ and frequency shifting $\alpha_{2, k}\left(\boldsymbol{g}_{j}^{\Omega_{i}}\right), j=1, \ldots, Q$ real coefficients are found, such that

$$
\begin{gathered}
\alpha_{1, k}\left(\boldsymbol{g}_{j}^{\Omega_{i}}\right) \mathbf{R}\left(s \alpha_{2, k}\left(\boldsymbol{g}_{j}^{\Omega_{i}}\right), \boldsymbol{g}_{k}^{\Omega_{i}}\right) \cong \mathbf{R}\left(s, g_{j}^{\Omega_{i}}\right), j \neq k \\
\alpha_{1, k}\left(\boldsymbol{g}_{j}^{\Omega_{i}}\right)=\alpha_{2, k}\left(\boldsymbol{g}_{j}^{\Omega_{i}}\right)=1, j=k
\end{gathered}
$$


When the response of the system under modeling needs to be computed in a specific design space point $\widehat{\boldsymbol{g}}$, a subdomain that contains $\widehat{\boldsymbol{g}}$ is to be found. For each vertex root macromodel of the found subdomain, the corresponding sets of scaling and frequency shifting coefficients $\alpha_{1, k}\left(\boldsymbol{g}_{j}^{\Omega_{i}}\right), \alpha_{2, k}\left(\boldsymbol{g}_{j}^{\Omega_{i}}\right)$ are interpolated in $\widehat{\boldsymbol{g}}$ and a rational model $\hat{\alpha}_{1, k} \mathbf{R}\left(s \hat{\alpha}_{2, k}, \boldsymbol{g}_{k}^{\Omega_{i}}\right)$ is built, where $\hat{\alpha}_{1, k}=\alpha_{1, k}(\widehat{\boldsymbol{g}})$ and $\hat{\alpha}_{2, k}=\alpha_{2, k}(\widehat{\boldsymbol{g}})$. Finally, the set of modified root macromodels $\hat{\alpha}_{1, k} \mathbf{R}\left(s \hat{\alpha}_{2, k}, \boldsymbol{g}_{k}^{\Omega_{i}}\right), k=1, \ldots, Q$, is interpolated at an input/output level as described in [8,9]. If a generic root macromodel $\mathbf{R}\left(s, \boldsymbol{g}_{k}^{\Omega_{i}}\right)$ has the state-space representation $\{\mathbf{A}, \mathbf{B}, \mathbf{C}, \mathbf{D}\}$, then a corresponding scaled and frequency shifted version $\hat{\alpha}_{1, k} \mathbf{R}\left(s \hat{\alpha}_{2, k}, \boldsymbol{g}_{k}^{\Omega_{i}}\right)$ has the state-space representation $\{\widetilde{\mathbf{A}}, \widetilde{\mathbf{B}}, \widetilde{\mathbf{C}}, \widetilde{\mathbf{D}}\}$ with

$$
\begin{aligned}
& \widetilde{\mathbf{A}}=\left(\hat{\alpha}_{2, k}\right)^{-1} \mathbf{A} \\
& \widetilde{\mathbf{B}}=\mathbf{B} \\
& \tilde{\mathbf{C}}=\hat{\alpha}_{1, k}\left(\hat{\alpha}_{2, k}\right)^{-1} \mathbf{C} \\
& \widetilde{\mathbf{D}}=\hat{\alpha}_{1, k} \mathbf{D}
\end{aligned}
$$

\subsection{Multivariate Interpolation}

Passivity is crucial when the macromodel is utilized in a circuit simulator (e.g. SPICE [20]) for transient analysis. Passive systems cannot generate more energy than they absorb through their electrical ports. When the system is terminated on any arbitrary passive loads, none of them will cause the system to become unstable [21]. The passivity of scattering input-output representations is also called nonexpansivity [22] A linear network described by scattering matrix $\mathbf{S}(s)$ is passive if [23]:

1. $\mathbf{S}\left(s^{*}\right)=\mathbf{S}^{*}(s)$ for all $s$, where " $*^{\text {" }}$ is the complex conjugate operator.

2. $\mathbf{S}(s)$ is analytic in $\mathfrak{R} e(s)>0$.

3. $\mathbf{I}-\mathbf{S}^{t}\left(s^{*}\right) \mathbf{S}(s) \geq 0 ; \forall s: \mathfrak{R} e(s)>0$.

Condition 3) for nonexpansivity is equivalent to the condition $\|\mathbf{R}(s)\|_{\infty} \leq 1$ ( $\mathbf{H}_{\infty}$ norm) [22], i.e., the largest singular value of $\mathbf{R}(s)$ does not exceed one in the right-half $s$-plane. The interpolated scaling and frequency shifting real coefficients $\alpha_{1, k}(\boldsymbol{g}), \alpha_{2, k}(\boldsymbol{g})$ have to satisfy the conditions

$$
\begin{array}{r}
0 \leq \alpha_{1, k}(g) \leq 1 \\
\alpha_{2, k}(g) \geq 0
\end{array}
$$

to guarantee the passivity of each root macromodel $\alpha_{1, k}(\boldsymbol{g}) \mathbf{R}\left(s \alpha_{2, k}(\boldsymbol{g}), \boldsymbol{g}_{k}^{\Omega_{i}}\right)$.

The coefficients $\alpha_{1, k}(\boldsymbol{g}), \alpha_{2, k}(\boldsymbol{g})$ are parameterized by positive multivariate interpolation schemes [24], which guarantee the passivity of each scaled and frequency shifted root macromodel over the entire design space by satisfying the properties (5a)-(5b). The same positive multivariate interpolation schemes are used to interpolate the set of modified root macromodels $\hat{\alpha}_{1, k} \mathbf{R}\left(s \hat{\alpha}_{2, k}, \boldsymbol{g}_{k}^{\Omega_{i}}\right), k=1, \ldots, Q$, at an input/output level, which results in a parametric macromodel, stable and passive over the entire design space.

In the bivariate case $(s, g)$, each interpolated function $\mathbf{T}(g)$ can be written as 


$$
\mathbf{T}(g)=\sum_{k=1}^{K_{1}} \mathbf{T}_{g_{k}} \ell_{k}(g)
$$

where $K_{1}$ represents the number of root macromodels and each interpolation kernel $\ell_{k}(g)$ is a scalar function satisfying the following constraints

$$
\begin{gathered}
0 \leq \ell_{k}(g) \leq 1 \\
\ell_{k}\left(g_{i}\right)=\delta_{k, i} \\
\sum_{k=1}^{K_{1}} \ell_{k}(g)=1 .
\end{gathered}
$$

A possible choice is to select $\ell_{k}(g)$ as in piecewise linear interpolation

$$
\begin{aligned}
& \frac{g-g_{k-1}}{g_{k}-g_{k-1}}, g \in\left[g_{k-1}, g_{k}\right], k=2, \ldots, K_{1}, \\
& \frac{g_{k+1}-g}{g_{k+1}-g_{k}}, g \in\left[g_{k}, g_{k+1}\right], k=1, \ldots, K_{1}-1, \\
& 0, \text { otherwise }
\end{aligned}
$$

In the general multivariate case, multivariate interpolation methods that belong to the general class of positive interpolation operators can be used, e.g., the piecewise multilinear and multivariate simplicial methods [17]. The computation of the interpolation kernel functions of these methods does not require the solution of a linear system to impose an interpolation constraint, since they only depend on the distribution of the estimation design space grid points. In the case of piecewise multilinear interpolation, each interpolated function $\mathbf{T}\left(g^{(1)}, \ldots, g^{(M)}\right)$ can be written as

$$
\mathbf{T}\left(g^{(1)}, \ldots, g^{(M)}\right)=\sum_{k_{1}=1}^{K_{1}} \cdots \sum_{k_{M}=1}^{K_{M}} \mathbf{T}_{\left(g_{k_{1}}^{(1)}, \ldots, g_{k_{M}}^{(M)}\right.} \ell_{k_{1}}\left(g^{(1)}\right) \cdots \ell_{k_{M}}\left(g^{(M)}\right)
$$

where each $\ell_{k_{i}}\left(g^{(i)}\right), i=1, \ldots, M$ satisfies constraints (7)-(9) and is selected as in piecewise linear interpolation. These positive interpolation schemes have been already used in $[8,9]$, where a parametric macromodel is built by interpolating a set of root macromodels treated as input-output systems, while preserving overall stability and passivity. The use of some interpolated scaling and frequency shifting system coefficients in the new presented technique allows to parameterize poles and residues indirectly, and hence it enhances the modeling capability of the proposed algorithm with respect to $[8,9]$, where the interpolation process were only applied to the root macromodels, and therefore only residues were parameterized.

\subsection{Passivity Preserving Interpolation}

Concerning the scaled and shifted root macromodels, a scaling coefficient $\alpha_{1}$ is applied at the input/output level of the system, while a frequency shifting coefficient $\alpha_{2}$ is a compression or expansion term for the Laplace variable $s$. It is easy to prove that if $\alpha_{2}$ satisfies (5b), passivity is preserved, and that if $\alpha_{1}$ satisfies (5a), the first two conditions for passivity are preserved. Concerning $\alpha_{1}$ and the third passivity condition 


$$
\left\|\alpha_{1} \mathbf{R}\left(\alpha_{2} s\right)\right\|_{\infty}=\alpha_{1}\left\|\mathbf{R}\left(\alpha_{2} s\right)\right\|_{\infty} \leq \alpha_{1} \leq 1
$$

Therefore, if $\alpha_{1}$ satisfies (5a), passivity is preserved. We have proven that passivity is preserved in the scaled and shifted root macromodels. Once a set of stable and passive scaled root macromodels is built, another interpolation step at an input/output level is performed to obtain a multivariate representation $\mathbf{R}(s, \boldsymbol{g})$. The proof of the passivity preserving interpolation of root macromodels at an input/output level can be found in $[8,9]$.

\section{Design Optimization}

Parametric macromodels can be used in an optimization process, where cost functions related to the frequency behavior of EM systems are involved. A general cost function can be represented as

$$
\mathbf{F}_{i}(\mathbf{g})=\mathbf{R}_{L i}-\mathbf{R}\left(s_{i}, \mathbf{g}\right)
$$

or

$$
\mathbf{F}_{i}(\mathbf{g})=\mathbf{R}\left(s_{i}, \mathbf{g}\right)-\mathbf{R}_{U i}
$$

with $i=1, \ldots, N_{S}$, where $N_{s}$ denotes the number of frequency samples, $\mathbf{R}_{L i}$ and $\mathbf{R}_{U i}$ represent lower and upper frequency response thresholds, respectively. A positive error value denotes that the specification is violated, while a negative error value indicates that the corresponding specification is satisfied. Several optimization algorithms can carry out the minimization of the cost functions (15)-(16). In the first example, we use a minimax optimization algorithm [25] that provides the optimum set of design parameter values $\tilde{\mathbf{g}}$

$$
\widetilde{\mathbf{g}}=\underset{\boldsymbol{g}}{\operatorname{argmin}}\left\{\max _{i}\left[\mathbf{F}_{i}(\mathbf{g})\right]\right\}
$$

In the second example, we optimize a cost function under some constraints using the Matlab [26] routine fmincon.

\section{Numerical results}

In what follows, two industrial design optimization examples are presented to validate the proposed method in industrial design processes. Let us define the absolute error

$$
\operatorname{Err}(\mathbf{g})=\max \left(\mid\left(R_{i, j}\left(s_{k}, \mathbf{g}\right)-H_{i, j}\left(s_{k}, \mathbf{g}\right) \mid\right)\right.
$$




$$
i=1, \ldots, P_{\text {in }}, \quad j=1, \ldots, P_{\text {out }}, \quad k=1, \ldots, K_{s}
$$

where $P_{\text {in }}$ and $P_{\text {out }}$ are the number of inputs and outputs of the system, respectively, and $K_{S}$ is equal to the number of frequency samples. The accuracy and quality of parametric macromodels are assessed using the worst case absolute error over the validation grid

$$
\begin{gathered}
\mathbf{g}_{\max }=\underset{\mathbf{g}}{\operatorname{argmax}} \operatorname{Err}(\mathbf{g}), \mathbf{g} \in \text { validation grid } \\
\qquad \operatorname{Err}_{\max }=\operatorname{Err}\left(\mathbf{g}_{\text {max }}\right)
\end{gathered}
$$

A bottom-up approach is used to adaptively select the number of poles for each root macromodel, so that the corresponding maximum absolute error is smaller than $-40 \mathrm{~dB}$.

\subsection{3-D example: SMA to PCB connection}

In this example, two SMA launch connecting a single ended stripline in a fourteen layer PCB are modeled and optimized. The trace is 549 mils long, 5 mils wide and 0.6 mils tick and it is routed on the fourth layer, in order to also consider the effect of the via stub related to the inner conductor of each SMA. The width $(W)$ of the trace and the radius $(R)$ of the seven grounding vias surrounding the SMA are used as parameters to optimize the performance of the launch. A waveguide port is used on the top of the SMA to excite a pure TEM mode in the structure, and therefore generate a meaningful scattering matrix. The dielectric material used for the PCB stack-up is Nelco with permittivity of $\varepsilon_{r}=3.5$ and loss tangent $\operatorname{tg}(\delta)=0.009$, whereas the metal is copper. Fig. 3 shows the structure under modeling. A trivariate parametric macromodel is built as a function of frequency, the width of the trace and the radius of the grounding vias (shown in Fig. 4). Table 1 shows their corresponding ranges.

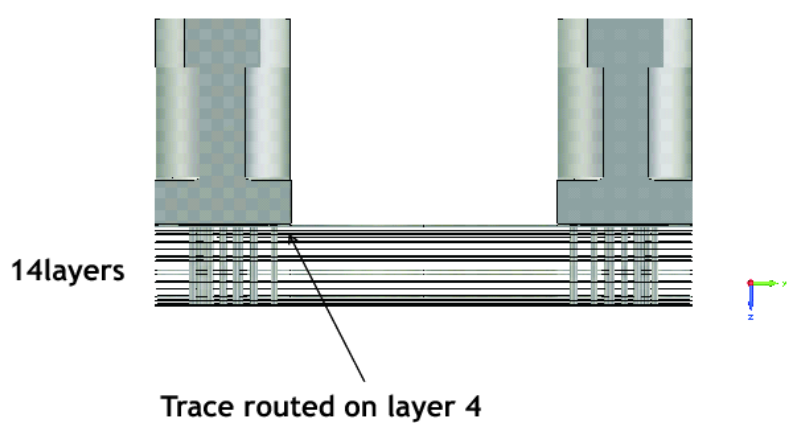

Figure 3: SMA to PCB connection. 


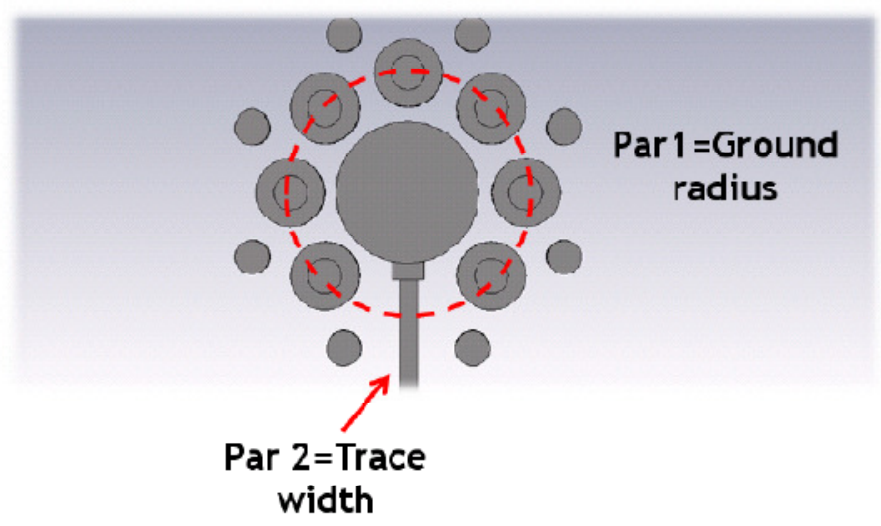

Figure 4: Design parameters of the SMA to PCB connection.

Table 1: Parameters of the SMA to PCB connection.

\begin{tabular}{|c|c|c|}
\hline Parameter & Min & Max \\
\hline $\begin{array}{c}\text { Frequency } \\
\text { (freq) }\end{array}$ & $0 \mathrm{~Hz}$ & $30 \mathrm{GHz}$ \\
\hline $\begin{array}{c}\text { Trace width } \\
\text { (W) }\end{array}$ & 3 mils & 34.5 mils \\
\hline $\begin{array}{c}\text { Vias Radius } \\
\text { (R) }\end{array}$ & 30.5 mils & \\
\hline
\end{tabular}

The scattering parameters have been computed by means of the commercial software [27] over the estimation grid ( 7 values of $W$ and 7 values of $R$ ) and the validation grid ( 6 values of $W$ and 6 values of $R$ ) considering 101 frequency samples. Each frequency-domain simulation (therefore each point in the estimation and validation design space grids) has taken 40 minutes. We have built root macromodels over the estimation grid by means of VF, each with an order chosen by the error-based bottom-up approach. Fig. 5 shows the distribution of the poles of the root macromodels in the Laplace domain and the corresponding influence of the design parameters. 


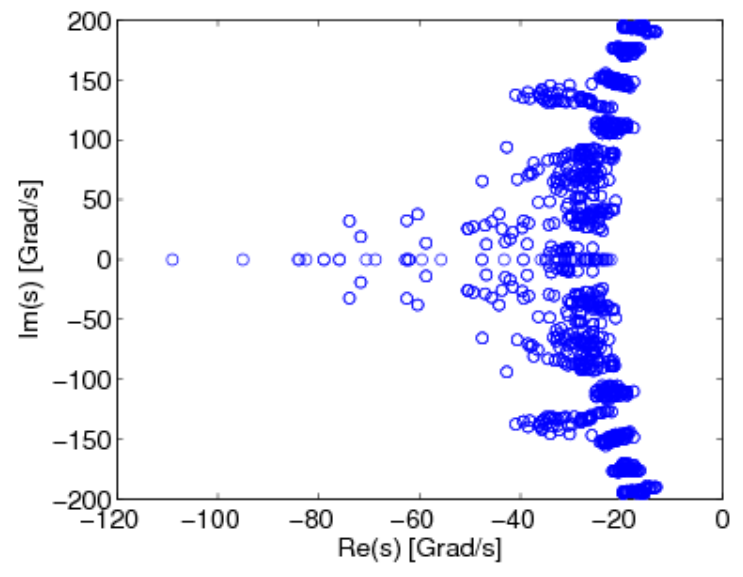

Figure 5: Poles of the root macromodels.

The CPU time needed to obtain the data samples from the EM solver [27] for the estimation and validation design space grids, and to build the parametric macromodel are shown in Table 2.

Table 2: CPU times model.

\begin{tabular}{|c|c|}
\hline Step & CPU time \\
\hline $\begin{array}{c}\text { Estimation } \\
\text { data }\end{array}$ & $32 \mathrm{~h} 40 \mathrm{~min}$ \\
\hline Validation data & $24 \mathrm{~h}$ \\
\hline $\begin{array}{c}\text { Parametric } \\
\text { macromodel }\end{array}$ & $4 \mathrm{~min} 23 \mathrm{~s}$ \\
\hline
\end{tabular}

Finally, a trivariate macromodel is obtained as explained in Section 2, using multilinear interpolation for the scaling, shifting coefficients and root macromodels. Figures 6-7 show the magnitude of the parametric macromodels of $\mathbf{S}_{11}(s, W, R)$ and $\mathbf{S}_{21}(s, W, R)$ for the trace width value $W=4$ mils and for the vias radius $R=32.5$ mils, respectively. Figure 8 compares $\mathbf{S}_{11}(s, W, R)$ and its macromodel for the values $R=\{30.83,32.16,33.5\}$ mils, $W=3.83$ mils that have not been used for the generation of the root macromodels. The worst case absolute error defined in (20) is equal to -35 $\mathrm{dB}$. 


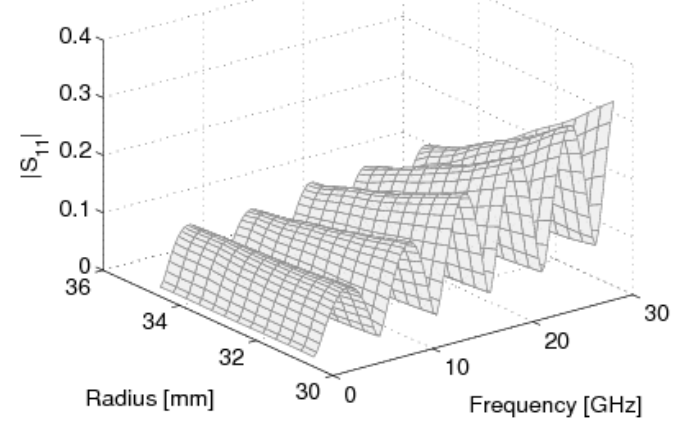

Figure 6: Magnitude of the trivariate macromodel of $\mathbf{S}_{11}(s, W, R)$ for $W=4$ mils.

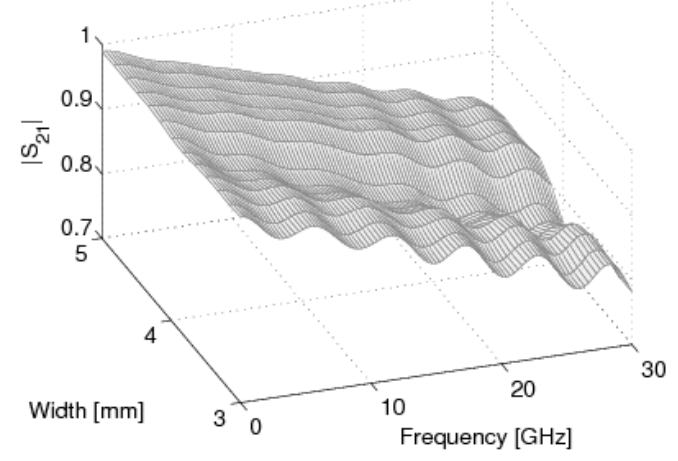

Figure 7: Magnitude of the trivariate macromodel of $\mathbf{S}_{21}(s, W, R)$ for $R=32.5$ mils.

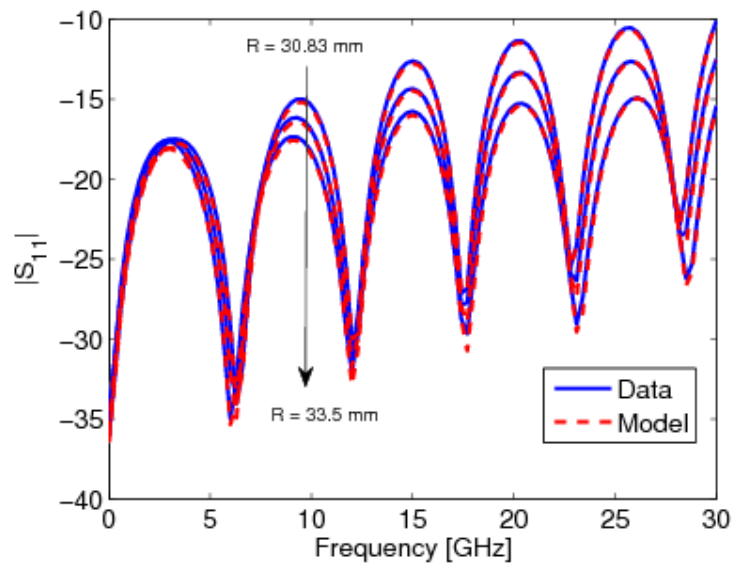

Figure 8: Magnitude of the trivariate macromodel of $\mathbf{S}_{11}(s, W, R)(R=\{30.83,32.16,33.5\}$ mils, $W=3.83$ mils). 
The behavior of the system is accurately modeled by the parametric macromodel, while stability and passivity are preserved over the entire design space. Then, the parametric macromodel has been used in an optimization process. The objective function is ensuring that the magnitude of $S_{11}$ is below $-20 \mathrm{~dB}$ in the bandwidth of interest. A minimax algorithm is used for the optimization. The starting values $\mathbf{g}_{\text {init }}=\{W, R\}=\{4$ mils, 32.5 mils $\}$ are used for the optimization and the optimal values are found to be $\tilde{\mathbf{g}}=\{W, R\}=\{4.94$ mils, 34.5 mils $\}$. Table 3 shows the computational time needed to perform the optimization by means of the commercial software [27] and the parametric macromodel.

Table 3: CPU time for the optimization (29 iterations).

\begin{tabular}{|c|c|}
\hline Method & CPU time \\
\hline CST Studio & $19 \mathrm{~h} \mathrm{20} \mathrm{min}$ \\
\hline $\begin{array}{c}\text { Parametric } \\
\text { macromodel }\end{array}$ & $4.4 \mathrm{~s}$ \\
\hline
\end{tabular}

As clearly seen, the obtained speed up confirms the applicability of the proposed parametric macromodeling method to accurate and efficient design optimizations of complex EM systems. Figure 9 shows the initial and optimized $\boldsymbol{S}_{11}$ response.

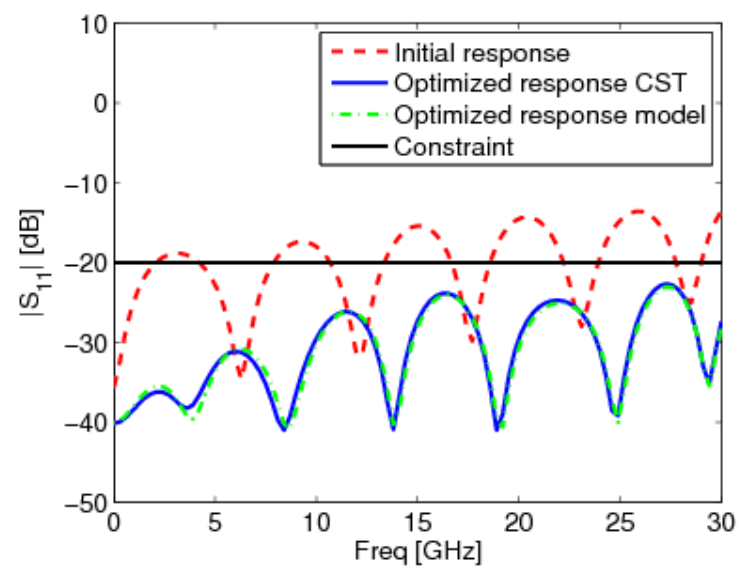

Figure 9: Optimization of $\mathbf{S}_{11}$ (starting values $\mathbf{g}_{\text {init }}=\{W, R\}=\{4$ mils, 32.5 mils $\}$, optimal values $\tilde{\mathbf{g}}=\{W, R\}=\{4.94$ mils, 34.5mils $\}$ ).

An initial set of simulations by means of [27] is required for the estimation and validation steps to build a parametric macromodel and therefore an initial computational effort, but once the parametric macromodel is created and validated, it becomes an accurate and efficient surrogate of the original system and can be used for each related design optimization or exploration in the design space defined during the construction of the parametric macromodel. Multiple uses of the parametric macromodel in design activities makes the initial computational effort negligible. 


\section{B. 3-D example: Mobile phone interconnection structure}

An interconnection structure used for a mobile phone application has been modeled in this example. The structure is shown in Fig. 10. It is composed of 10 lines and an etched ground plane. A trivariate parametric macromodel is built as a function of frequency, the spacing between the first two lines at the center of the structure and the angle of etching. The input and output ports are numbered as shown in Fig. 10. Table IV shows their corresponding ranges.

Table 4: Parameters of the mobile phone flex interconnection.

\begin{tabular}{|c|c|c|}
\hline Parameter & Min & Max \\
\hline $\begin{array}{c}\text { Frequency } \\
\text { (freq) }\end{array}$ & $\mathrm{OHz}$ & $20 \mathrm{GHz}$ \\
\hline Spacing $(S)$ & $25 \mu \mathrm{m}$ & $65 \mu \mathrm{m}$ \\
\hline Angle $(\alpha)$ & $45^{\circ}$ & $65^{\circ}$ \\
\hline
\end{tabular}
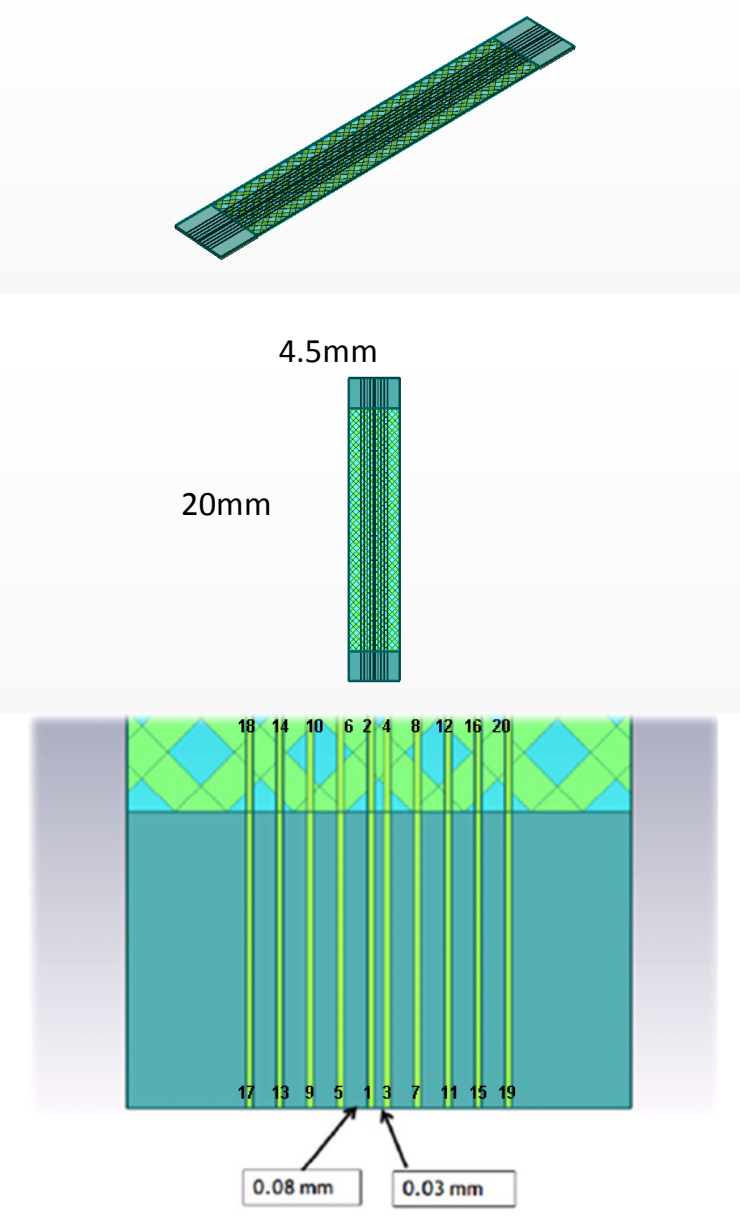

Figure 10: Mobile phone flex interconnection. 
The scattering parameters have been computed by means of the commercial software [27] over the estimation grid ( 5 values of $S$ and 4 values of $\alpha$ ) and the validation grid ( 4 values of $S$ and 3 values of $\alpha$ ) considering 101 frequency samples. Each frequency-domain simulation (therefore each point in the estimation and validation design space grids) has taken 30 minutes. We have built root macromodels over the estimation grid by means of VF, each with an order chosen by the error-based bottom-up approach. Figure 11 shows the distribution of the poles of the root macromodels in the Laplace domain and the corresponding influence of the design parameters. The CPU time needed to obtain the data samples from the EM solver [27] for the estimation and validation design space grids, and to build the parametric macromodel are shown in Table 5.

Table 5: CPU times model.

\begin{tabular}{|c|c|}
\hline Step & CPU time \\
\hline $\begin{array}{c}\text { Estimation } \\
\text { data }\end{array}$ & $10 \mathrm{~h}$ \\
\hline Validation data & $6 \mathrm{~h}$ \\
\hline $\begin{array}{c}\text { Parametric } \\
\text { macromodel }\end{array}$ & $7 \mathrm{~min} 14 \mathrm{~s}$ \\
\hline
\end{tabular}

As in the previous example, a trivariate macromodel is obtained as explained in Section II, using multilinear interpolation for the scaling, shifting coefficients and root macromodels. Figures 1213 show the magnitude of the parametric macromodels of $S_{11}(s, S, \alpha)$ and $S_{13}(s, S, \alpha)$ for the spacing $S=$ $45 \mu \mathrm{m}$ and for the angle etching $\alpha=55^{\circ}$, respectively. Fig. 14 compares $S_{13}(s, S, \alpha)$ and its macromodel for the values $S=\{30,60\} \mu \mathrm{m}, \alpha=55^{\circ}$ that have not been used for the generation of the root macromodels. The worst case absolute error defined in (20) is equal to $-35 \mathrm{~dB}$.

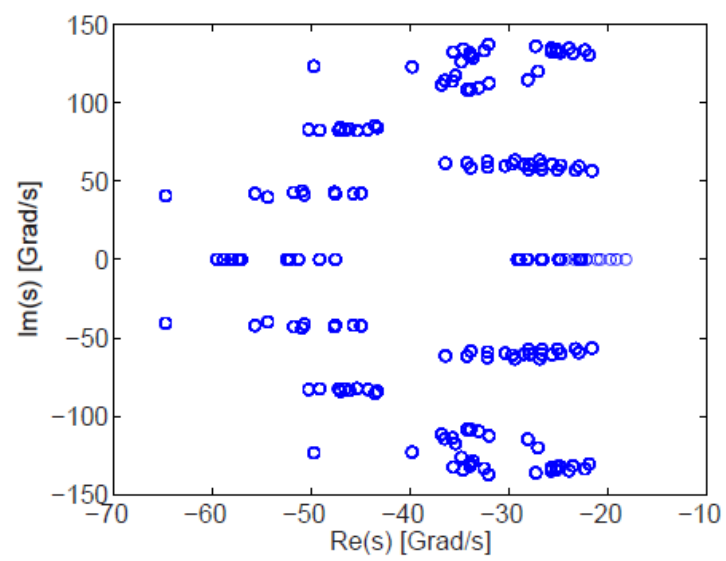

Figure 11: Poles of the root macromodels 


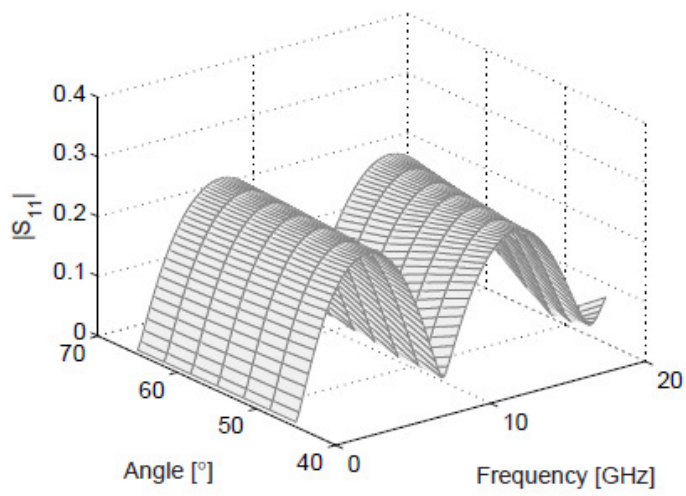

Figure 12: Magnitude of the trivariate macromodel of $S_{11}(s, S, \alpha)$ for $S=45 \mu \mathrm{m}$.

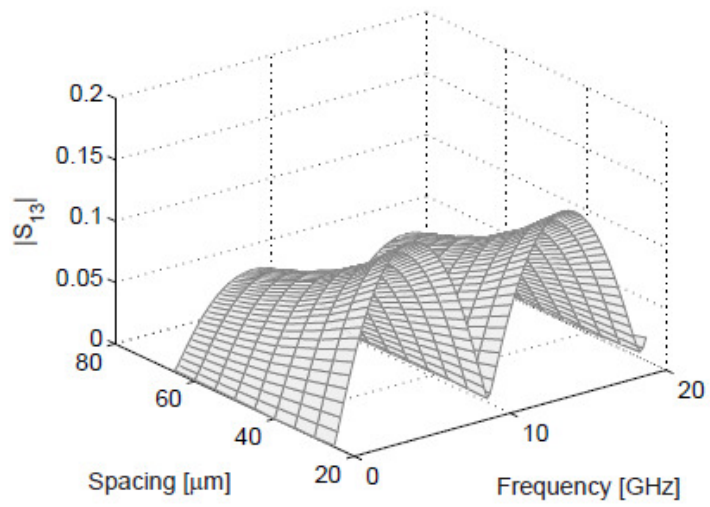

Figure 13: Magnitude of the trivariate macromodel of $S_{13}(s, S, \alpha)$ for $\alpha=55^{\circ}$.

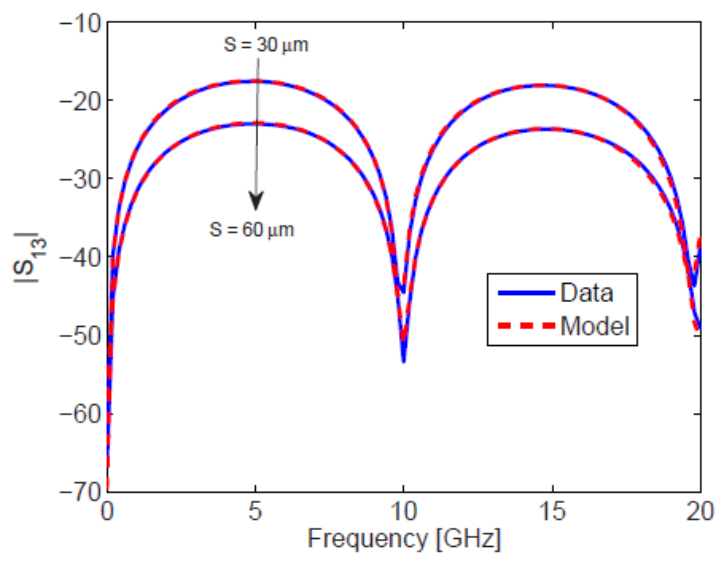

Figure 14: Magnitude of the trivariate macromodel of $S_{13}(s, S, \alpha)(S=\{30,60\} \mu \mathrm{m}$ and $\alpha=55^{\circ}$ ).

The parametric macromodel is able to accurately describe the behavior of the system, while 
guaranteeing stability and passivity over the entire design space. The parametric macromodel has been used in an optimization process, where the cost function is ensuring that the magnitude of $S_{13}$ is below $-22 \mathrm{~dB}$ in the bandwidth of interest under the constraints of minimizing the values of the optimum $S$ and $\alpha$. The Matlab fmincon algorithm is used for the optimization. The starting values $\mathbf{g}_{\text {init }}$ $=\{S, \alpha\}=\left\{31.5 \mu \mathrm{m}, 58^{\circ}\right\}$ are used for the optimization and the optimal values are found to be $\tilde{\mathbf{g}}=\{S, \alpha\}$ $=\left\{54 \mu \mathrm{m}, 50^{\circ}\right\}$. Table 6 shows the computational time needed to perform the optimization by means of the commercial software [27] and the parametric macromodel.

Table 6: CPU time for the optimization (21 iterations).

\begin{tabular}{|c|c|}
\hline Method & CPU time \\
\hline CST Studio & $10 \mathrm{~h} 30 \mathrm{~min}$ \\
\hline $\begin{array}{c}\text { Parametric } \\
\text { macromodel }\end{array}$ & $46 \mathrm{~s}$ \\
\hline
\end{tabular}

The proposed parametric macromodeling technique is able to perform accurate and efficient design optimizations of complex EM systems. Fig. 15 shows the initial and optimized $\mathrm{S}_{13}$ response.

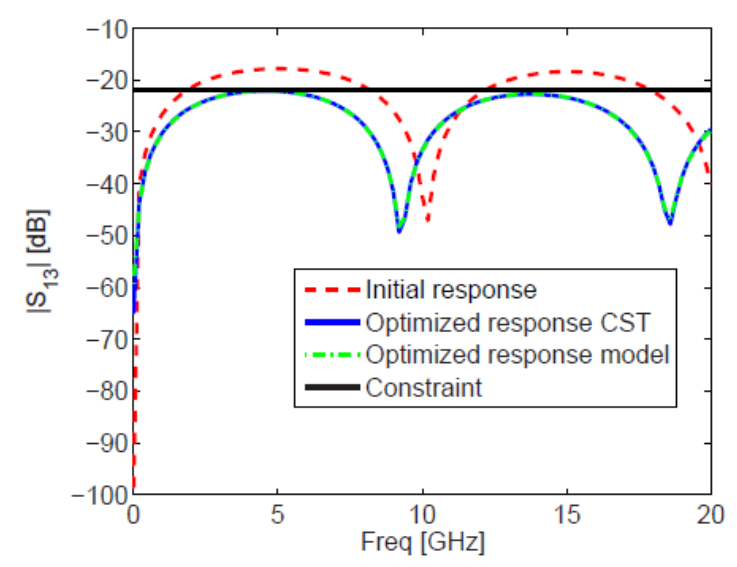

Figure 15: Optimization of $S_{13}$

The parametric macromodel has also been used in another optimization process, where the cost function is ensuring that the magnitude of $S_{14}$ is below $-30 \mathrm{~dB}$ in the bandwidth of interest under the constraints of minimizing the values of the optimum $S$ and $\alpha$. The Matlab fmincon algorithm is used for the optimization. The starting values $\mathbf{g}_{\text {init }}=\{S, \alpha\}=\left\{45 \mu \mathrm{m}, 55^{\circ}\right\}$ are used for the optimization and the optimal values are found to be $\tilde{\mathbf{g}}=\{S, \alpha\}=\left\{39.5 \mu \mathrm{m}, 45^{\circ}\right\}$. Table 7 shows the computational time needed to perform the optimization by means of the commercial software [27] and the parametric macromodel. 
Table 7: CPU time for the optimization (27 iterations).

\begin{tabular}{|c|c|}
\hline Method & CPU time \\
\hline CST Studio & $13 \mathrm{~h} 30 \mathrm{~min}$ \\
\hline $\begin{array}{c}\text { Parametric } \\
\text { macromodel }\end{array}$ & $53 \mathrm{~s}$ \\
\hline
\end{tabular}

Figure 16 shows the initial and optimized $\mathrm{S}_{14}$ response.

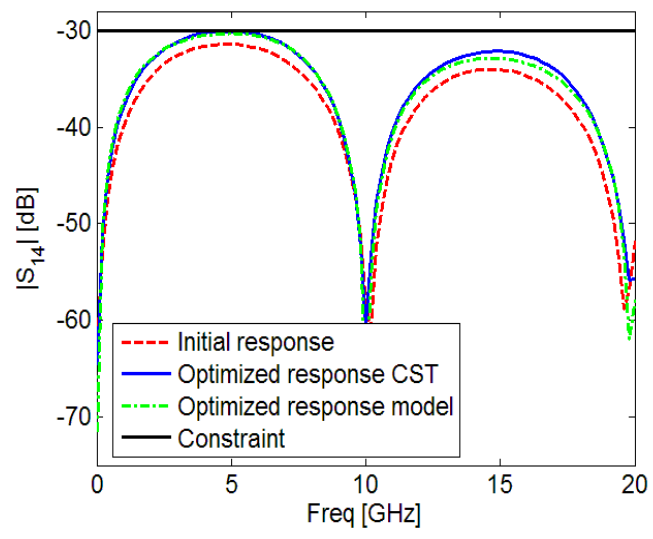

Figure 16: Optimization of $S_{14}$

As in the previous example, we note that multiple uses of the parametric macromodel in design activities makes the initial computational effort to estimate and validate the model negligible.

\section{Conclusions}

We have proposed a new parametric macromodeling technique for systems described by scattering representations, which is able to indirectly parameterize both poles and residues, while ensuring overall stability and passivity. It is based on an efficient and reliable combination of rational identification, a procedure to find scaling and frequency shifting system coefficients, and positive interpolation schemes. The proposed method is used in the optimization process of two complex electromagnetic systems, which shows the speedups obtained using parametric macromodels instead of EM simulations and its applicability to a real design process flow. 


\section{ACKNOWLEDGMENTS}

This work was supported by the Research Foundation Flanders (FWO).

\section{REFERENCES}

[1] I. Couckuyt, F. Declercq, T. Dhaene, H. Rogier and L. Knockaert, "Surrogate-based infill optimization applied to electromagnetic problems", International Journal of RF and Microwave Computer-Aided Engineering, vol. 20, no. 5, pp. 492-501, Sept. 2010.

[2] Virender K. Sadhir, Inder J. Bahl and David A. Willems, "Cad compatible accurate models of microwave passive lumped elements for mmic applications", International Journal of Microwave and Millimeter-Wave Computer-Aided Engineering, vol. 4, no.2, pp. 148-162, April 1994.

[3] E. Ragonese, A. Scuderi, Tonio Biondi and G. Palmisano, "Scalable lumped modeling of single-ended and differential inductors for RF IC design", International Journal of RF and Microwave Computer-Aided Engineering, vol. 19, no 1, pp 110-119, Jan. 2009.

[4] M. Schoeman and P. Meyer, "Comparative study on adaptive rational macromodels of highly resonant structures", International Journal of RF and Microwave Computer-Aided Engineering, vol. 17, no. 3, pp. 286-294, May 2007.

[5] Q-J Zhang, F. Wang and M. Nakhla, "Optimization of high-speed VLSI interconnects: A review", International Journal of RF and Microwave Computer-Aided Engineering, vol. 7, no. 1, pp. 83-107, Jan. 1997.

[6] D. Deschrijver, T. Dhaene and D. De Zutter, "Robust parametric macromodeling using multivariate orthonormal vector fitting," IEEE Trans. Microw. Theory Tech., vol. 56, no. 7, pp. 1661-1667, Jul. 2008.

[7] P. Triverio, S. Grivet-Talocia, M. S. Nakhla, “An improved fitting algorithm for parametric macromodeling from tabulated data," in Proc. in Workshop Signal Propagation on Interconnects, Avignon, France, May 2008, pp. 1-4.

[8] F. Ferranti, L. Knockaert, and T. Dhaene, "Guaranteed passive parameterized admittancebased macromodeling," IEEE Trans. Adv. Packag., vol. 33, no. 3, pp. 623-629, Aug. 2010.

[9] - - "Parameterized S-parameter based macromodeling with guaranteed passivity," IEEE Microw. Wireless Compon. Lett., vol. 19, no. 10, pp. 608-610, Oct. 2009.

[10] F. Ferranti, L. Knockaert, T. Dhaene, and G. Antonini, "Passivity preserving parametric macromodeling for highly dynamic tabulated data based on Lur'e equations," IEEE Trans. Microw. Theory Tech., vol. 58, no. 12, pp. 3688 -3696, Dec. 2010.

[11] P. Triverio, M. Nakhla, and S. Grivet-Talocia, "Extraction of parametric circuit models from scattering parameters of passive RF components," in European Microwave Conference (EuMC), Sept. 2010, pp. $1635-1638$.

[12] J. De Caigny, J. F. Camino, and J. Swevers, "Interpolating model identification for SISO linear parameter-varying systems," Mechanical Systems and Signal Processing, vol. 23, no. 8, pp. $2395-2417,2009$.

[13] G. Balas, R. Chiang, A. Packard, and M. Safonov, "Robust Control Toolbox User's Guide, Version 3," The Mathworks, Inc., 2005.

[14] B. Gustavsen and A. Semlyen, "Rational approximation of frequency domain responses by vector fitting," IEEE Trans. Power Delivery, vol. 14, no. 3, pp. 1052-1061, Jul. 1999.

[15] D. Saraswat, R. Achar and M. Nakhla, "On passivity enforcement for macromodels of S- 
parameter based tabulated subnetworks," in Proc. Of the IEEE Int. Symp. on Circuits and Systems, vol. 4, May 2005, pp. 3777-3780.

[16] T. Dhaene, D. Deschrijver, and N. Stevens, "Efficient algorithm for passivity enforcement of S-parameter based macromodels," IEEE Trans.Microw. Theory Tech., vol. 57, no. 2, pp. 415420, Feb. 2009.

[17] W. A. Weiser and S. E. Zarantonello, "A note on piecewise linear and multilinear table interpolation in many dimensions," Mathematics of Computation, vol. 50, no. 181, pp. 253264, Jan. 1988.

[18] H. W. Kuhn, "Some combinatorial lemmas in topology," IBM Journal of Research and Development, vol. 4, no. 5, pp. 518-524, 1960.

[19] D. F. Watson, "Computing the n-dimensional Delaunay tessellation with application to Voronoi polytopes," The Computer Journal, vol. 24, no. 2, pp. 167-172, Febr. 1981.

[20] L. W. Nagel, "SPICE: A computer program to simulate semiconductor circuits," University of California, Berkeley, Electr. Res. Lab. Report ERL M520, May 1975.

[21] E. A. Guillemin, Synthesis of Passive Networks. John Wiley and Sons, New York, 1957.

[22] S. Boyd, L. El Ghaoui, E. Feron and V. Balakrishnan, Linear Matrix Inequalities in System and Control Theory. Philadelphia, PA: SIAM,1994, vol. 15.

[23] B. D. Anderson, S. Vongpanitlerd, Network Analysis and Synthesis. Englewood Cliffs, NJ: Prentice-Hall, 1973.

[24] G. Allasia, "Simultaneous interpolation and approximation by a class of multivariate positive operators," Numerical Algorithms, vol. 34, no. 2, pp. 147-158, Dec. 2003.

[25] D.-Z. Du and P. M. Pardalos, Minimax and Applications. Kluwer Academic Publishers, 1995.

[26] “Matlab User's Guide," The Mathworks, Inc., Natick, 2009.

[27] CST MICROWAVESTUDIO, www.cst.com. 